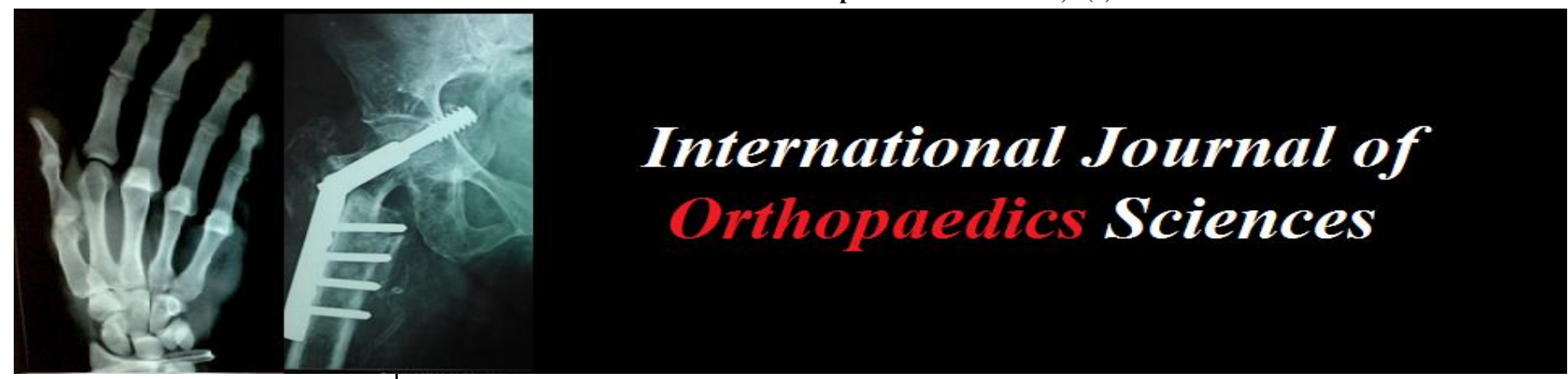

E-ISSN: 2395-1958

P-ISSN: 2706-6630

IJOS 2021; 7(1): 605-608

(C) 2021 IJOS

www.orthopaper.com

Received: 09-11-2020

Accepted: 15-12-2020

Vijay Mahantesh Sunkad Assistant Professor, Assistant Professor, Department of Orthopedics, KIMS, Koppal,

Karnataka, India

Nagadurga Prasanna Reddy N Senior Resident, Department of Orthopedics, KIMS, Koppal,

Karnataka, India
Corresponding Author: Nagadurga Prasanna Reddy N Senior Resident, Department of Orthopedics, KIMS, Koppal,

Karnataka, India

\section{Effectiveness of surgical management of lumber intervertebral disc prolapse}

\author{
Vijay Mahantesh Sunkad and Nagadurga Prasanna Reddy N
}

DOI: https://doi.org/10.22271/ortho.2021.v7.i1j.2547

\section{Abstract}

Background: Low back ache is the commonest musculoskeletal problem of the mankind. Disc prolapse is one of the most common and important cause of low back ache. Stress \& strain on the back is more because of the bipedal nature which leads to mechanical failure of the disc architecture and is maximally exerted over L4-L5 level.

Objectives: The need of this study to evaluate the results of laminectomy alone or laminectomy with foramenectomy or laminectomy with discectomy depending on pre-operative evaluation, with regard to patients post-operative subjective evaluation with ODI scoring system and its complications.

Method: A prospective study was carried out on 30 cases of lumbar disc prolapse came to the Department of Orthopaedics, NMC\&RC Raichur after through clinical evaluation. The data were collected using detailed clinical proforma, clinical examination, required investigations, pre-operative, post-operative \& Follow up 1,3,6 months) assessment, pre and post-operative assessment focused on pain SLRT, Neurological assessment, spinal movements, occupational function and ODI score.

Results: The findings of the study reveals that 80 percent $(n=24)$ subjects showed excellent improvement, another 20 percent $(n=6)$ subjects showed good improvement and no subjects showed poor improvement after the proper surgical treatment by comparing through ODI scoring

Conclusion: The results demonstrated that, proper evaluation of the patients with accuracy of level and involvement of disc pathology before operative procedure with appropriate selection of surgery relieves the symptoms and restore the function of patients with least complications and disabilities.

Keywords: Low back ache, Disc prolapsed, laminectomy, laminectomy with foramenectomy, laminectomy with discectomy

\section{Introduction}

Low back pain (LBP) which was known as an ancient curse is now known as a modern international epidemic. Epidemiological data shows that the prevalence of LBP is not decreasing and is still at epidemic proportions. It is the most widely reported musculoskeletal disorder in the world, and 70-80\% of all people will develop LBP in their life.

In industrialized countries, approximately $50-80 \%$ of the adult populations have low back pain at some time in their lives. There are various causes of Low Back Pain, although there are many structural causes for low back pain like injury to the supporting paraspinal muscles, the complex network of supporting ligaments, facet joint cartilage, vertebral bones and compression of neuronal structures due to herniated nucleus pulposus of lumbar disci out of which the lumbar disc herniation is one of the most frequent reason for physical, functional restriction in patients. From the experience of many years, the beneficial role of surgery in degenerative spine disease of the lumbar spine is a major point of discussion'. By making effective efforts the role of surgery for degenerative spine disease is understood. The promotion of realistic expectations and development of pre-screening tools to assist with patient selection procedure has prompted the search for risk factors'. The determinants of surgical outcomes have been identified by numerous studies over the last 10-15 years". The findings of the results are either on the basis of some of the retrospective ${ }^{[1]}$ or prospective researches ${ }^{[2]}$. Retrospective researches have formed the basis of a number of resulting events". There have been reports that higher success rate (70-95\%) achieved in case of surgical treatment of symptomatic lumbar disc herniation with degenerative spine disease, assessed through authenticate outcome scores and patient approvall" [3]. 
In addition, patients who have undergone surgical treatment are found to possess increased short term outcome instead of conservative treated patients". The comparative results of patients treated with surgery and conservatively treated patients have revealed that surgical treatment is much better at short-term follow-up (up to 1 year) however no variations have been showed among treatments at long-term follow-up". Still, it has been found that patients who had undergone surgical treatment had experienced fast pain relief, improvement of function and satisfaction in comparison to conservative patients". In spite of major technical successes of the different operative procedures for various disorders it is established that some patients operated for spine disease are still left with poor results ${ }^{[4]}$.

Numerous outcome measures have been adopted and these measures range from limits which offer a numerical score to physical and psychosocial elements, for example, the low back outcome score ${ }^{[5]}$. The Oswestry Disability Index ${ }^{[6]}$ was used for easy categorization of methods which includes good, fair and poor. The "Visual Analog Scale" (VAS) used to measure the severity of pain is employed to visualize the suffering of the patient during patient's clinical care. This tool was introduced in medical science by Clarke and Spear (1964) in order to assess patient's health ${ }^{[7]}$.

The variable character of low back pain, its multiplicity of cause and the difficulties in its treatment renders this affection. One of the most perplexing and also one of the most frequent problems that confront an orthopaedic surgeon. Impairments of the back and spine are ranked as the most frequent cause of limitation of activity in people younger than 45 years by the National Center for Health Statistics ${ }^{[8]}$. Lumbar disc prolapse as an important cause of backache has been recognized long ago. The lumbar spinal column during its development into a secondary curvature that is the lumbar lordosis has become adapted to transmit and support enormous biomechanical loads resulting from transference of the body weight of the trunk down through the pelvis into the lower extremities. It also provides mobility of flexion, extension, lateral flexion, rotation and posture ${ }^{[9]}$.

Intervertebral disc disease and disc hemiation are most prominent in otherwise healthy people in the 3rd and 4th decades of life. It accounts for a majority of cases of low backache seen by an orthopaedician in clinical practice and is a major contributor of functional disability ${ }^{[8]}$. In 1934, Mixter and Barr published their study which concluded that laminectomy with decompression and extraction of herniated lumbar disc could improve suffering caused by sciatic pain. Open discectomy is now the "gold standard" for operative intervention in patients with herniated lumbar disc whose conservative treatment has failed ${ }^{[9]}$.

With the basic understanding of disease process, new diagnostic techniques, refinements in surgical techniques, improvements in surgical and instrumentation have made the surgical removal of the offending disc herniation a reasonably safe procedure with majority of satisfactory results. Thus the present study tried to analyze the efficacy of this procedure in Navodaya Medical College and Research Centre, Raichur and comparing it with the available studies in the literature.

\section{Methods}

The present prospective study includes 30 cases of lumbar disc prolapse treated during the period of May 2011 to September 2013 in KIMS Hospital, Bangalore. Patient assessment was done in the OPD and wards and findings noted in the clinical proforma made for the study. The patients underwent radiological investigations (MRI) to confirm the diagnosis and to know the level of lesion.

\section{Source of data}

All cases above 18 years of age with lumbar inter vertebral disc prolapse were admitted at KIMS hospital, Bangalore and those who met the inclusion and the exclusion criteria (as given below) during the study. All patients were treated surgically by open discectomy.

\section{Inclusion criteria}

1. Lin-relieved pain radiating along the course of the nerve in the lower limb respectively.

2. Nerve tension signs - positive

3. Associated neurological deficits

4. Confirmed by MRI scanning

5. Above 18 yrs of age.

Exclusion Criteria: Thoroco- lumbar injuries, Lumbar canal stenosis, Spondylolisthesis, Fail back syndrome, Medically unlit for surgery, Peripheral neuropathy, Infective conditions.

\section{And Tumors (neoplastic) lesion}

Method: All patients admitted at Navodaya Medical College Hospital and Research Center, Raichur those who meet the inclusion and exclusion criteria and ODI score more than $65 \%$ were taken up with history and clinical examination.

Patients with signs and symptoms of disc prolapse, ODI $>65 \%$, MRI showing conclusive disc prolapse and who come under the inclusion criteria were selected and admitted.

1. Investigations required for surgery was done.

2. A pre anaesthetic evaluation was done.

3. Pre-operative preparations were performed and informed written consent was taken.

4. Methods such as laminectomy alone, or with foremenotomy or discectectomy was chosen according to the pre-operative evaluation.

5. Follow ups 1, 3 and 6 months were made.

Technical terminology concepts used in the report Operative procedure: Posterior mid central incision Anaesthesia: General anaesthesia

Position: Prone position

Prophylactic antibiotics used were a third generation cephalosporin just before the operation and continued after surgery upto 5 days.

The placement of incision was determined by the help of image intensifier Noting the level of iliac crest (highest point) corresponding to L4 spinous process.

The length of the incision for a single level laminectomy was usually $3-6 \mathrm{~cm}$.

With a scalpel, divide longitudinally the ligament between the two spinous processes in the most distal part of the wound.

Insert a small, blunt periosteal elevator through this opening so that its end rests on the junction of the spinous process with the lamina of the more proximal vertebra. Move the handle of the elevator proximally and laterally to place under tension the muscles attached to this spinous process.

With a scalpel moving from distal to proximal, strip the muscles subperiosteally from the lateral surface of the process.

Place the end of the elevator in the wound so that its end rests on the junction of the spinous process with the lamina of the next most proximal vertebra, and repeat the procedure as described. Repeat the procedure until the desired number of 
vertebrae have been exposed. For operations requiring exposure of both sides of the spine, use the same technique on each side.

This approach exposes the spinous processes and medial part of the laminae.

Pack each segment with a tape sponge immediately after exposure to lessen bleeding. Divide the supraspinous ligament precisely over the tip of the spinous processes, and denude subperiosteally the sides of the processes because this route leads through a relatively avascular field; otherwise, the arterial supply to the muscles is encountered.

Blood loss can be decreased further by using electrocautery and a suction apparatus. Replace blood as it is lost.

Expose the spinous processes from distal to proximal as just described because the muscles can then be stripped from the spinous processes in the acute angle between their insertions and the bone.

If exposure in the opposite direction is attempted, the knife blade or periosteal elevator tends to follow the direction of the fibers into the muscle and divides the vessels, increasing hemorrhage.

Self-retaining retractors were put to improve the field of vision. The level of the spinous process was then confirmed with the help of intraoperative fluoroscopy. The spinous process was removed and the ligamentum flavum beneath the caudal aspect of lamina was retracted. The structures were clearly exposed. The laminae were carefully nibbled and ligament flavum removed with the help of a kerrison rongeur. After this the dura was clearly visible. After the dura was exposed adequately inspection of the nerve root was done. A thin instrument like a ducal retractor was used displaces the anterior surface of nerve root dura from the floor of spinal canal. The nerve root was retracted medially to visualize the underlying disc. It was usually seen as a bulging posterior longitudinal ligament or as an extruded fragment. If frank extrusion was encountered an effort was made to remove it early in the direction of nerve to avoid retraction on the nerve root. If frank extrusion was not encountered posterior longitudinal ligament was carefully examined for any defect. A cruciate incision was made by second knife over the annulus and posterior longitudinal ligament and the disc fragments were removed piecemeal with the help of disc forceps. Disc space is irrigated with saline; disc excision with disc forceps is repeated. Bleeding is controlled by keeping blood pressure below $400 \mathrm{rnm} \mathrm{Hg}$ and using cottonoid patties. After this the nerve root is traced up to the intervertebral foramen and root canal decompression is done at the intervertebral foramen. Root reexamined and was made to move with minimum force. The free movement of nerve signified that the procedure was complete.

\section{Wound closure}

After removing the disc material and making the nerve root free, the exposed dura surface was covered with gel foam Wound is closed in layers, keeping the vacuum suction drain.

\section{After care}

Postoperative IV antibiotics were given for a period of 5 days and later continued orally until suture removal. Allowed only log roll movements on bed, with active hip, knee and ankle movements. Isometric abdominal and lower extremity exercises started immediately after surgey depending upon the patients' tolerance and reaction of the wound. The drain was removed and patient allowed sitting with the lumbar corset on 3rd post-operative day. Post-operative analgesia was continued on as per required basis and stopped after 4' postoperative day. Patients' spine flexion exercises started and allowed to walk with lumbar corset on the 5th post-operative day. Sutures were removed on 12 ' post-operative day and spine extension exercises were allowed. During this period they were told to avoid bending, sitting for prolonged periods, straining, lifting heavy weight and usage of western commode for defecation till 12 weeks.

\section{Result}

$40 \%$ of my study patients are between the age group in between 20 to 30 years old. $23.3 \%$ in $31-40$ years age group, $26.7 \%$ in $41-50$ years age group and $10 \%$ in 50 to 55 years age group respectively.

Study reveals majority of the patients are fall into 20-30 years age group of about $40 \%$. $60 \%(n=18)$ of my subjects were males and $40 \%(n=12)$ were females. out of total number of patients $50 \%$ of them were farmers, $16.7 \%$ were drivers, $10 \%$ were housewifes, $6.7 \%$ were labour, $3.3 \%$ were clerk, $3.3 \%$ were tailor, $6.7 \%$ were atheles and $3.3 \%$ were students.

Level of compression occurs $56.7 \%$ at L4-L5 and $43.3 \%$ at L5-S1 level and most common site of compression is at L4L5 compare to other levels. Table 1

Table 1: Distribution of study patients based on level of compression $\mathrm{n}=30$

\begin{tabular}{|c|c|c|}
\hline Level of compression & No. Of cases & Percentage \\
\hline L4 - 15 & 17 & 56.7 \\
\hline L5 -s1 & 13 & 43.3 \\
\hline Total & 30 & 100 \\
\hline
\end{tabular}

Duration of illness before they got operated i.e more 6months in $53.33 \%$ of my study patients, upto 3-6 months in $26.67 \%$ patients, upto 1-3 months in $20 \%$ patients. The study reveals that more than $50 \%$ of the patients are suffered from symptoms for more than 6 months before surgery. Table 2

Table 2: Distribution of the study patients based on the duration of illness before surgery

\begin{tabular}{|c|c|c|}
\hline Duration of illness (months) & No. Of cases & Percentage \\
\hline$<1$ & 0 & 0 \\
\hline $1-3$ & 6 & 20 \\
\hline $3-6$ & 8 & 26.67 \\
\hline$>6$ & 16 & 53.33 \\
\hline Total & 30 & 100 \\
\hline
\end{tabular}

$73.33 \%$ of my patients are presented with bilateral radicular pain compared to unilateral radicular pain $26.67 \%$. 57\% of my patients are right sided radiculopathy more compared with left side $43 \%$ which includes unilateral and bilateral radiculopathy [complains of right side radiculopathy more then the left side] patients.

Table 3: Distribution of the study patients based on the duration of conservative management before surgery

\begin{tabular}{|c|c|c|}
\hline $\begin{array}{c}\text { Conservative management (Before } \\
\text { Operation) }\end{array}$ & No. Of cases & Percentage \\
\hline$<1$ month & 0 & 0 \\
\hline $1-3$ months & 8 & 26.27 \\
\hline $3-6$ months & 10 & 33.33 \\
\hline$>6$ months & 12 & 40 \\
\hline TOTAL & 30 & 100 \\
\hline
\end{tabular}

Study reveals the patients undergoing surgery are all straight leg raising test and lasegues tests are positive.

Patients have $43.33 \%$ of paracentraldiscal defect compare to central disc defect 23.33. Table 4. 
Table 4: Distribution of study patients based on side of intervertebral disc bulge

\begin{tabular}{|c|c|c|}
\hline Quadrants of IVDP & No. of cases & Percentage \\
\hline Central & 7 & 23.33 \\
\hline Para central & 13 & 43.33 \\
\hline Foraminal & 10 & 33.33 \\
\hline Total & 30 & 100 \\
\hline
\end{tabular}

Patient's undergone laminectomy with discectomy $23.33 \%$ and laminectomy with foramenotomy $33.33 \%$ compared to only laminectomy $43.33 \%$.

Study reveals laminectomy alone gives desire results, with discectomy and foramenotomy at appropriate patients when needed. Table 5.

Table 5: Distribution of study patients based on surgery undergone

\begin{tabular}{|c|c|c|}
\hline Operations & No. Of cases & Percentage \\
\hline Laminectomy with discectomy & 13 & 43.33 \\
\hline Laminectomy with foramenotomy & 10 & 33.33 \\
\hline Laminectomy & 7 & 23.33 \\
\hline Total & 30 & 100 \\
\hline \multicolumn{2}{|c}{}
\end{tabular}

\section{Discussion}

Males are more prone for stress and strainful activities like heavy weight lifting, prolong sitting in their daily activities, and may be the strong relation to more prone to suffers from intervertebral disc prolapsed.

\begin{tabular}{|c|c|c|}
\hline Study & Gender & Percentage \\
\hline Present study & Male & $66 \%$ \\
\hline S. Sharma and Shankar ${ }^{[10]}$ & Male & $76 \%$ \\
\hline
\end{tabular}

Since the young age group 20-40 years who are exposed to more work and stressful activities, may be the strong relation to more prone to suffers from intervertebral disc prolapsed, The maximum amount of movement occurs at the L4-L5 level hence it is subjected to more stress and repeated trauma which leads to degeneration and has an end result of IVDP

\begin{tabular}{|c|c|c|}
\hline Study & Level of disc space & Percentage \\
\hline Present study & L4 & $56.7 \%$ \\
\hline Chan WB Peng and William YEO & L4 & $66.7 \%$ \\
\hline
\end{tabular}

Most of subjects in the Present study showed right sided postero-lateral disc prolapse therefore involving the right lower limb

\begin{tabular}{|c|c|c|}
\hline Study & Side of Sciatic Pain & Percentage \\
\hline Present Study & Right & $57 \%$ \\
\hline Righesso $^{[11]}$, Orlando MD (2007) & Left & $63 \%$ \\
\hline
\end{tabular}

Open disc excision under direct vision offers sufficient adequate exposure for lumbar disc excision with smaller incision, lesser morbidity, shorter convalescence and hence the lesser complication rate.

\begin{tabular}{|c|c|c|}
\hline Study & Method & Percentage: Excellent \\
\hline Present Study & Open Discectomy & $80 \%$ \\
\hline Richard Davis ${ }^{[12]}$ & Open Discectomy & $89 \%$ \\
\hline Pappes et al. ${ }^{[13]}$ & Open Discectomy & $77.6 \%$ \\
\hline
\end{tabular}

\section{Conclusion}

As we are blessed with so many sophisticated instruments to approach, handling soft tissue, dural, nerve roots and with direct vision we can achieve fewer complications with good outcome to a greater extent compared to before. The results demonstrated that, proper evaluation of the patients with accuracy of level and involvement of disc pathology before operative procedure with appropriate selection of surgery relieves the symptoms and restore the function of patients with least complications and disabilities.

Open procedures are easy to perform, more understanding of pathology all around, economical, with least complications and the most effective means of treating lumbar disc prolapse. So open procedures are still the "Gold Standard" in operative management of lumbar Intervertebral disc prolapse for Indian scenario.

Acknowledgements: I would like to express my profound gratitude to all my patients, for their cooperation and faith, without them it would be impossible to complete my study.

\section{Declarations \\ Funding: None}

\section{Conflict of interest: None}

Ethical approval: Permission for the study was obtained from the College authorities prior to commencement.

\section{References}

1. Pappas CTE, Harrington T, Sonntag VKH. Outcome analysis in 654 surgically treated lumbar disc herniation. Neurosurgery 1992;30(6):862-6.

2. Stambough JL. Lumbar disc herniation: an analysis of 175 surgically treated cases. Journal of Spinal Disorders \&Techniques 1997;10(6):488-92.

3. Andrew J Schoenfeld and Bradley K Weiner. Treatment of lumbar disc herniation: Evidence-based practice. Available from: http: //www.ncbi.nlm.nih.gov /pmc /articles / PMC2915533/

4. Fritz B, Hagg 0, Jonsson B. One-year report from the Swedish national Stromqvist B spine register. Swedish society of spinal surgeons. Acta Orthopaedica: Supplementum 2005;76(319):1-24.

5. Greenough CG, Fraser RD. Assessment of outcome in patients with low back pain. Spine 1992;17(1):36-41.

6. Fairbank JC, Cooper, Davies JII, O'Brien JP. The Oswestry low hack questionnaire. Physiotherapy 668 pain disability 1980;66(8):271-3.

7. Vsladdell G. Evaluation of results in lumbar spine surgery. Acta Orthopacdica Scand: Supplementum 1993;251(164):134-7.

8. Wood George W. Lower back pain and disorders of intervertibcral disc. Chapter 39, Cambell's Operative orthoaedics, Edn, cdt, Canalc S. Terry, Missouri, Mosby 2003, 1955-2029.

9. Mixter WJ, JS Barr. Rupture of the intervertebral disc with involvement of the spinal canal. New England Journal of Medicine 1934;211:210-215.

10. Sharma S, Sankaran B. A clinical profile of prolapsed lumbar intervertebral disc and its management. Indian Journal Orthopaedics 1980;14(2):204-212.

11. Righesso 0, Falavigna A, Avanzi 0. Comparison of open discectomy with microendoscopic discectomy in lumbar disc herniations: results of a randomized controlled trial. Neurosurgery 2007;61:545.

12. Richard Snell S. Anatomy of the back Chap-12, Clinical anatomy of the medical students 5th edition, Edt Richard S. Snell, Boston Little Brown and Company 1995, 823828.

13. Pappas CT, Harrington $\mathrm{T}$, Sonntag VK. Outcome analysis in 645 surgically treated lumber disc herniation. Neurosurgery 1993;32(5):879. 